\title{
Karin Kutter
}

\section{Ölreserven und Ölinteressen. Wie glaubwürdig sind die Angaben zu den weltweiten Ölreserven?}

\section{Unterbewertung und Überbewertung der Ölreserven}

Für das gegenwärtige kapitalistische Wirtschaftssystem gibt es wohl keinen wichtigeren Rohstoff als Öl, was nicht zuletzt die jüngste Runde der Ölpreissteigerungen und die damit verbundenen Befürchtungen für die weitere Entwicklung der Weltwirtschaft deutlich gemacht haben. Der Ölverbrauch nimmt weltweit immer weiter zu, während die Ölreserven endlich und nicht erneuerbar sind. Damit wird die Frage nach dem Umfang der Ölreserven zu einem Politikum ersten Ranges. „Everybody hates this topic but the oil industry hates it more than anybody else" (Schindler/Zittel 2000: 3) konstatiert Colin Campbell, Gründer der ASPO (Association for the Study of Peak Oil), ${ }^{1}$ auf die Frage nach den Ölreserven. Jedes Jahr werden zu diesem Thema verschiedene Studien veröffentlicht, wic die des United States Geological Survey (USGS) oder die der Internationalen Energieagentur oder des Konzerns ExxonMobil. Die bei weitem wichtigste und einflussreichste Studie dürfte allerdings der BP Statistical Review of World Energy Report sein, der hier im Mittelpunkt stehen soll. Dieser Bericht listet nicht nur die Reserve-, Produltions- und Verbrauchsangaben von $\ddot{\mathrm{O}}$, sondern

1 Colin J. Campbell war jahrzehntelang in leitender Position bei großen Ölfirmen tätig. Die ASPO ist ein Netzwerk aus Wissenschaftlern, welches das Maximum und die künftige Abnahme der Ölproduktion zu bestimmen versucht. auch von Erdgas und Kohle, sowie die Verbrauchszahlen von Atomenergie und Hydroenergie auf. Doch wie glaubwürdig sind diese Reserveangaben?

Als Datenquelle der Studie werden unter anderem die Zeitschrift World Oil, und das $O i l$ and Gas Journal angegeben. Letztere befragt alljährlich die staatlichen und privaten Ölfurmen nach ihren Fördermengen und den Reserven. Doch den einzelnen Firmen bleibt es dabei selbst überlassen, welche Zahlen sie angeben, denn die Qualität der Daten wird von niemandem überprüft.

Und genau hier liegt das erste Problem. Denn die Angaben zu den Ölreserven werden in der Auseinandersetzung zwischen den Ländern der OPEC und dem Rest der Welt häufig als Mittel der Propaganda benutzt. Viele OPEC-Staaten befürchten, dass die von ihnen abhängigen Industriestaaten entweder auf Alternativen umsteigen oder ihre Suche nach Öl woanders verstärken könnten. „Daher wird ein 'Propagandakrieg' gefuihrt mit der Botschaft, man habe noch so viel Öl, dass man die Industriestaaten jederzeit mit billigem $\ddot{\mathrm{O} l}$ überschwemmen könne, sobald diese selbst nach teurerem Öl suchen oder auf Alternativen ausweichen. Dann würden diese Investitionen wertlos" (Campbell et al. 2003: 183). Die Nicht-OPEC-Staaten wollen hingegen Glauben machen, es gäbe noch genügend Öl auf der Welt, auf das man zurück greifen könnte, „sobald die OPEC ihre Stellung missbrauchen würde" (Schindler/Zittel 2000: 18). So sind die Reserve-Angaben in der BP Statistical Review of World Energy 2004 mit Vor- 
sicht zu genießen. Denn beide Seiten, sowohl die OPEC als auch die NichtOPEC-Mitglieder sind ,damit beschäftigt, sich gegenseitig Märchen zu erzählen" (Schindler/Zittel 2000: 18).

Dieses Märchen-Erzählen wird auch innerhalb der OPEC praktiziert. Denn die Größe der Reserven eines Landes spielt eine wichtige Rolle bei den Verhandlungen um die Förderquoten. Wer größere Reserven hat, kann bei der vereinbarten Gesamtförderung leichter eine höhere Quote durchsetzen. Daher gibt es gute Gründe, den Angaben der OPEC Länder, nach denen sich ihre Reserven in den 1980er Jahren um ganz erhebliche Beträge - nämlich zwischen 42 und 197 Prozent - erhöht hätten, zu misstrauen (Campell/Laherrère 1998). Die BP-Studie hinterfragt solche gigantischen Zuwächse der Ölreserven jedoch nicht, sondern nimmt sie einfach als gegeben hin.

Ölfirmen hingegen neigen eher zu einer Unterbewertung ihrer Reserven. So werden von Anfang an niedrigere Angaben zu Neufunden gemacht, um dann Schritt für Schritt die Angaben über Reserven zu erhöhen. Begründet wird eine solche Erhöhung meist mit einer verbesserten Technik der Fördermethoden. Diese Praxis spart nicht nur Steuern ein. Den Investmentgesellschaften, die für die Ölfirmen als Aktienkäufer wichtig sind, wird damit ein Bild des kontinuierlichen Wachstums suggeriert. Doch inzwischen ist der durch die Unterbewertung der Ölreserven entstandene Puffer weitestgehend aufgebraucht. Eine Steigerung der Reserveangaben allein durch eine Neubewertung bereits gefundener Ölfelder wird immer schwieriger. Auch zeigt sich zunehmend, dass die jetzigen Neufunde, die nur noch kleine Ölvorkommen beinhalten, überbewertet werden. Die Ölfirmen nutzen also bei den jährlichen Angaben, die sie dem Oil \& Gas Journal zur Verfügung stellen, einen sehr großen Spielraum aus.

Bisweilen kommen diese Tricksereien der Ölfirmen jedoch ans Tageslicht. So musste der Shell-Konzern im Januar die- sen Jahres seine Reserven ura rund 3,9 Milliarden Barrel (1 Barrel sind 159 Liter) nach unten korrigieren. Im März erfolgte dann nochmals eine Korrektur um weitere 470 Millionen Barrel. „Damit entpuppten sich nun mehr als 20 Prozent der weltweiten Reserven von Shell als falsch verbucht" (Kroder 2004). Während die Wertanpassung im Januar auf Fehleinschätzungen von Reserven in Australien und Nigeria zurückzuführen war, waren für die emeute Korrelktur im März falsche Daten für das OrmenLange-Feld vor der norwegischen Küste die Ursache. Laut Colin Campbell sind diese Falschangaben symptomatisch für die gesamte Branche (siehe das Interview bei Kroder 2004).

Dabei sind insbesondere die Daten über die sogenannten gesicherten Reserven von Ende 2003 über den Nahen Osten in der BP-Studie äußerst zweifelhaft. Sie betragen insgesamt 726,2 Mrd. Barrel. Glaubt man Matt Simmons, einem führenden Energieanalysten und Gründer von Simmons \& Company International, könnte dieser „conventional energy wisdom: Middle East oil is limitless" (Simmons 2004: 2ff) bald fatale Folgen haben, da ,all long-term oil demand/supply models" annähmen, „Middle East oil can grow as fast as oil demand rises (...), if more is needed drill anywhere" (Simmons 2004: 2ff).

Saudi Arabien nimmt dabei die Rolle des Ölkönigs ein. Hier liegen 25 Prozent der als gesichert geltenden Reserven weltweit. Nur sieben Schlüsselfelder produzieren jedoch über 90 Prozent des saudi-arabischen Öls. Das bedeutet, dass ein Produktionsausfall eines dieser Ölfelder nicht einfach durch die verstärkte Nutzung anderer Ölfelder ersetzt werden kann. Simmons ließ eine zwölf Monate dauernde Studie über die saudiarabischen Ölfelder durchführen und kam zu dem Ergebnis, dass in SaudiArabiens gigantischen Ölfeldern das Maximum der Ölförderung vielleicht schon erreicht sei und eine schnelle Abnahme der Fördermengen eventuell schon in 
den nächsten drei Jahren bevorsteht (Schempt 2004). Nur durch aggressives Einpumpen von Wasser bleibe das Produktionsniveau noch so hoch (Simmons 2004: 22). Durch dieses Einpumpen wird der Druck im Inneren des Ölfeldes erhöht und durch die so entstandene Überaktivität kann kurzfristig der Produktionsrückgang gestoppt werden. Colin Campbell sieht die Lage in SaudiArabien weniger drastisch, er glaubt, dass das Fördermaximum erst im nächsten Jahrzehnt erreicht wird (Ruppert 2004). Doch dass Saudi Arabiens Reserveangaben falsch sind, weiß auch er. Stichhaltige Informationen liefern nur ehemalige Insider. In einem Interview verweist Campbell auf den Ex-Chef des staatlichen Ölkonzerns Saudi Aramco, der sich als „Ex“ frei genug fühlte, um zu reden und gegenüber dem Oil and Gas Journal die gesichert erschlossenen Reserven Saudi-Arabiens auf $130 \mathrm{Mrd}$. Barrel bezifferte (Darley 2004). Zum Vergleich: In der aktuellen BP-Studie werden für Saudi Arabien 262,7 Mrd. Barrel als gesicherte Reserven angegeben - das ist mehr als doppelt so viel.

\section{Konventionelles und unkonventionelles Öl}

Doch wie kann die BP-Studie zu solch hohen Angaben über die Ölreserven kommen? Ein Hinweis darauf findet sich im Kleingedruckten der Studie: „This year`s Statistical Review includes new improved data series for proved oil (...) reserves. The new series does not necessarily meet the United States Securities and Exchange Commissions definitions and guidelines for determining proved reserves" (BP 2004: 4). Unter dem Begriff "Öl" fasst BP sowohl "Canadian oil sands under active development, (...) gas condensate and natural gas liquids as well as oil" (BP 2004: 4) zusammen. Auch fehlt eine Unterscheidung zwischen „regular oil, deepwater, polar [and] heavy oil" (BP 2004: 4). Diese Unterscheidung wäre aber äußerst notwendig, „because each [kind of oil] has ist own costs, characteristics and, above all, depletion profile (Campbell 2004a).

Tiefseeöl und Polaröl sowie Schweröl, Ölsand, Flüssiggas und Kondensat zählt man dabei zu den sogenannten unkonventionellen Erdölreserven. „Ökonomen unterscheiden konventionelles und unkonventionelles Öl gerne (...) über den Preis. Danach wird das teuer zu erschließende Öl als unkonventionell bezeichnet" (Campbell et al. 2003: 89). Diese Definition korreliert natürlich auch mit den schwierigen Produktionsbedingungen von unkonventionellem Öl. Durch den höheren Aufwand bei der Förderung werden so auch die Produktionskosten höher.

Ein Beispiel für die schwierigen Produktionsbedingungen ist das $\ddot{O} l$, das man in der Tiefsee findet. Behördenstatistiken definieren Tiefseeöl ab einer Meerestiefe von 200 oder 300 Metern, während Firmenstatistiken die Grenze eher bei 500 oder 600 Metern ansetzen. Dieses Öl ist oft mit Paraffin angereichert, und das hat Konsequenzen: „Sobald das Öl mit dem kalten Meerwasser in den Pipelines am Boden in Kontakt kommt, verfestigt sich das Paraffin" (ebd.: 103). Die Pipelines müssen deshalb beheizt und isoliert werden, was zusätzliche Kosten verursacht. Das Bohren in diesen sehr großen Tiefen ist durch viele technische Innovationen inzwischen kein Problem mehr, allerdings ist es schwierig, das Nebenprodukt Gas zu entsorgen. Teilweise pumpt man das Gas auch wieder zurück ins Ölfeld, um dort den Druck zu erhöhen. „Lohnend ist also folglich nur die Förderung aus sehr großen Feldern, doch davon gibt es nicht sehr viele" (ebd.: 100).

Wenig lohnend ist auch die Förderung des sogenannten Polaröls, das aus Öllagerstätten nördlich und südlich des 66 . Breitengrades gewonnen wird. In diesen eiskalten Wüsten ist die Ölförderung mit weitaus größeren Schwierigkeiten verbunden, als in warmen Gegenden. Auch verbergen sich in den Polargebieten keine „erstklassigen Ölvorkommen" (ebd.: 102). Probleme bringen auch die Schweröle 
mit sich. Sie sind zwar bezüglich ihrer petrochemischen Eigenschaften mit dem Rohöl vergleichbar, doch unterscheiden sie sich in ihrer Dichte und der Viskosität. Dadurch wird die Fließfähigkeit bestimmt, weshalb Schweröle mit sehr viel mehr Aufwand als normales Rohöl aus dem Gestein gelöst werden müssen. Hier spielt also wieder der Kostenfaktor eine entscheidende Rolle.

Teersande schließlich sind Sandsteine, die sehr zähflüssige Kohlenwasserstoffe beinhalten. Man rechnet sie ebenfalls zu den Schwerölen. Im westkanadischen Fort McMurray könnten davon über 2500 Mrd. Barrel vorhanden sein. Das wäre mehr als das gesamte abbaubare Vorkommen an konventionellem Öl auf der Erde (Campbell et. al. 2003: 90) - eigentlich ein Milliardengeschäft. Doch die Förderung des Ölsandes ist sehr aufwändig und damit äußerst kostspielig. Erst die hohen Weltmarktpreise haben diese Art von $\ddot{O} 1$ überhaupt konkurrenzfähig gemacht. In Kanada herrscht folglich "Goldgräberstimmung", da die Technologie um Öl aus Ölsand zu fördern in den letzten zwanzig Jahren erheblich verbessert wurde. Allerdings hat der Ölrausch in Kanada auch seine Schattenseiten. So berichtet der Bürgermeister von Fort McMurray, Douglas L. Faulkner, „Ich habe gehört, dass wir in unserer Gemeinde eine Zahl von Menschen mit Asthma-Problemen, Lungen-Problemen und Atembeschwerden haben. Ich weiß noch nicht, ob das mit dem ÖlsandGeschäft zusammenhängt" (NDR 2004). Doch ist dies wohl der Fall, denn in der Gegend um Fort McMurray entstehen etwa ein Viertel aller kanadischen Abgase (ebd.). Auch der Abraum, der in riesigen Schuttbergen zurückbleibt, ist stark belastet. Wenn der Abbau von Teersanden noch gesteigert wird, könnte das fatale Folgen für die Umwelt haben. „Sollten eines Tages etwa 10 Prozent der heutigen Weltölproduktion aus dieser Gegend [um Fort McMurray] kommen, dann wären das etwa 8 Millionen Barrel Öl täglich - oder anders ausgedrückt, jeden
Tag würden etwa 4-5 Cheopspyramiden aus Abraummaterial (...) angehäuft werden, jedes Jahr wären das etwa $1600 \mathrm{Py}^{-}$ ramiden!" (Campbell et al. 2003: 92).

Es sollte nun deutlich geworden sein, wie wichtig die Unterscheidung zwischen konventionellem und unkonventionellem Öl ist. Statistiken und Hochrechnungen, die die Unterschiede der Fördermöglichkeiten ignorieren und einfach Mengen (einschließlich den Mengen an unkonventionellen Öl) zusammenrechnen, können daher leicht zu dem Ergebnis kommen, dass die Ölproduktion auch künftig weiter steigen wird. Von dieser fragwürdigen Möglichkeit wird in der BP-Studie ausgiebig Gebrauch gemacht. Für Kanada sieht die BP-Studie einen Zuwachs der Reserven um rund 76 Prozent innerhalb der letzten zwanzig Jahre. Über 200 Prozent Zuwachs weist die Studie für Venezuela aus. Doch das meiste Öl, das in Venezuela gefördert werden kann, ist unkonventionelles Öl. Und für Angola, wo Tiefseeöl gefördert wird, wachsen die Angaben innerhalb von zwanzig Jahren sogar um über 400 Prozent!

\section{Die ASPO-Studie}

Aufgrund dieser zweifelhaften Bewertungen hat die ASPO eine neue Studie erstellt. Ihre Bewertungen ,refer to best estimates of what actually remains to be produced from known fields, assuming maximum economic incentive and the application of known technology" (Campbell 2004a). Außerdem bezieht sie sich auf "Regular Oil, which includes condensate, but excludes heavy, deep water, polar, and NGL ${ }^{2}$ from gasfields" (ebd.). Während BP 1147,7 Mrd. Barrel an weltweiten Ölreserven angibt, kommt die ASPO nur auf einen Wert von $780 \mathrm{Mrd}$. Barrel. Insgesamt also ein Unterschied von ungefähr $368 \mathrm{Mrd}$.

2 Natural Gas Liquids (Ethan, Butan, Isobutan, Propan, etc.), schwere Bestandteile des Erdgases, die bei leichtem Druck und Umgebungstemperatur flüssig werden. 
Barrel, allerdings gibt es erhebliche Differenzen bei einzelnen Ländern. Für Kanada sinken hier die Reserven von 16,9 Mrd. Barrel (nach der BP-Studie) auf lediglich 5,8 Mrd. Barrel (nach ASPO). Für Venezuela ergibt sich ein Verlust von 43,4 Mrd. Barrel und in Angola verringern sich die angegebenen Zahlen ron ursprünglich 8,9 auf 3,9 Mrd. Barrel. Auch im Nahen Osten sind die Diskrepanzen zwischen der BPStudie und den ASPO-Angaben sehr groß. BP hatte nämlich die offiziellen Zahlen der Länder des Nahen Ostens einfach akzeptiert, obwohl diese Angaben seit vielen Jahren nahezu unverändert geblieben sind, was angesichts der hohen Fördermengen völlig unplausibel ist (ebd.). In Saudi-Arabien sieht die ASPO zum Beispiel nur ein Potenzial von $144 \mathrm{Mrd}$. Barrel, während die BPStatistik 262,7 Mrd. Barrel veranschlagt. Mit 59,9 Mrd. Barrel findet die ASPO im Iran weniger als die Hälfte der Reserven, welche die BP Statistik angibt (130,7 Mrd. Barrel). Die Angaben über dic Reserven des Nahen Ostens sind von immenser Bedeutung, liegen doch hier, nach der aktuellen BP-Studie, 63,3 Prozent aller weltweiten Ölreserven. Eine Überbewertung gerade in dieser Region könnte fatale Folgen haben.

\section{Das Maximum der Ölförderung als Bestimmungsfaktor der Öl- produktion}

Auch muss berücksichtigt werden, dass die aktuelle Verfügbarkeit von Erdöl von den Produktionsmöglichkeiten und damit insbesondere vom Produktionsmaximum bestimmt wird. Angaben über theoretisch vorhandene Reserven, wie sie in der BP Studie zu funden sind, sagen damit nichts über die tatsächliche Verfügbarkeit von Erdöl aus (Schindler/Zittel 2000: 11).

Die Ölförderung eines jeden Ölfeldes folgt aufgrund der geologischen Gegebenheiten dem Verlauf einer Glockenkurve. Anfangs sorgt der hohe Druck im Ölfeld für eine relativ leichte Produkti- on, durch den Zubau von weiteren Fördersonden kann dic Produktion ausgcweitet werden. Wenn etwa die Hälfte des Öls im Reservoir entnommen ist, ist der Druck im Ölfeld so weit abgesunken, dass die Zähigkeit des Öls das Förderprofil $\mathrm{zu}$ bestimmen beginnt. Damit ist das Fördermaximum erreicht. Ab diesem Zeitpunkt sinkt die Entnahmerate aus dem Ölfeld. Durch künstliche Erhöhung des Drucks, zum Beispiel durch die Injektion von Wasser oder Erdgas, kann die Produktion jenseits des Maximums zwar noch beeinflusst werden, doch werden, so Schindler/Zittel (2000: 12), derartige Möglichkeiten der Produktionssteigerung weithin überschätzt. Davon abgesehen werden durch solche Maßnahmen keine neuen Reserven erschlossen, das vorhandene Öl wird lediglich schneller gefördert.

Campbell et al. (2003: 74) kommen daher zu dem Ergebnis, dass die Angaben über die Ölreserven, die von der BP-Studie gemacht werden, „eher irreführend" sind. Denn „entscheidend für strukturelle Änderungen der Energieversorgung ist nicht die (statistische oder dynamische) Reichweite der Reserven, also 'wie lange reicht das Öl bei vorgegebener jährlicher Förderquote?" sondern die Frage: $\mathrm{Ab}$ welchem Zeitpunkt kann die Ölproduktion aus geologischen, technischen und ökonomischen Gründen nicht mehr erhöht werden, sondern nimmt tendenziell nur

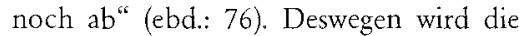
Debatte um das Maximum der Ölförderung ,the next big thing" (Williams 2004) werden. Einige Kommentatoren verwenden sogar die Formel „Peak oil = Economic Hiroshima" (Savinar 2004). Wann genau dieser „Peak point" bei der Ölförderung eintritt, ist aufgrund der unsicheren Datenlage und den Interessen der einzelnen Unternehmen und der jeweiligen Länder das Ergebnis in die eine oder andere Richtung zu verschieben, sehr ungewiss (Kopytoff 2004). Colin Campbell sieht den Höhepunkt der Ölförderung in seinen neuesten Berechnungen in Jahr 2008 (Campbell 2004b). 
Diese Prognose bezeichnet Leonardo Maugeri, Topmanager der italienischen Öl firma Eni (Agip), allerdings als einen „Witz" (Friebe 2004): „Campbell hat vor Jahren für 1989 den Peak vorausgesagt, dann für 1995, dann für 1998, dann für 2002" (Friebe 2004). Campbell kommentiert seine immer wieder von neuem korrigierten Aussagen so: „Manche sagen, ich bin gescheitert. Ich sage: Ich mache Fortschritte." (Friebe 2004) Doch es gibt noch mehr Stimmen gegen Campbell. So zeichnet die „U.S. Energy Information Administration" ein anderes Bild der Situation. Sie sieht das Maximum der Ölförderung in einem Zeitraum zwischen 2021 und 2112 , so dass es anscheinend keinen Grund zu übertriebener Sorge gibt.

Ein Vergleich der von der BP-Studie angegebenen Ölreserven von 2002 und 2003 zeigt allerdings, dass doch Grund zur Sorge besteht. Zwar weist die BP-Statistik insgesamt einen Zuwachs von 1,4 Milliarden Barrel für das Jahr 2003 aus, doch muss man dieses Ergebnis etwas detaillierter betrachten. Für Nordamerika und den Nahen Osten werden geringe Rückgänge der Reserven verzeichnet. In Südund Zentralamerika wird jedoch eine Erhöhung der Reserven für das Jahr 2003 festgestellt, doch kommt dieses Plus ausgerechnet von Brasilien und Venezuela, die für ihre Tiefsee- bzw. Schwerölvorkommen bekannt sind. Auch im asiatischen Raum können nur Dank der Ölschiefervorkommen und Gasquellen Australiens die Reserven erhöht werden. Für die Reservezuwächse in Europa und Eurasien ist allein Russland verantwortlich. Erhofft man etwa in den unerforschten Polarschelfs von Russland Öl zu finden?

Die Reserveangaben sind also jetzt schon äußerst bedenklich. Wann genau das $\mathrm{Ma}$ ximum der Ölförderung erreicht sein wird, kann niemand sagen, doch dass es in den kommenden Jahren sein wird, lässt sich kaum bezweifeln. $\mathrm{Ob}$ Campbells Aussage dieses Mal stimmt, wird man erst rückblickend beurteilen können, denn schließlich sieht man „einen Gipfel nicht, wenn man auf ihm steht ${ }^{\text {s }}$ (Kopytoff 2004).
Auch Matt Savinar, Autor des Buches The Oil Age is over, sieht der Zukunft der Ölförderung beunruhigt entgegen: "The term 'peak' tends to suggest a nice, neat curve. But in the real world, the landing will not be soft. As we hit the peak, soaring prices - $\$ 70, \$ 80$ even $\$ 100$ a barrel - will encourage oil companies and oil states to scour the planet for oil. (...) This post-peak production will deplete remaining reserves all the more quickly, thus ensuring that the eventual decline is far steeper and more sudden. As one US government geologist put it (...) recently, "the edge of a plateau looks like a cliff.' As production falls of this cliff, prices won 't simply increase; they will fly" (Kopytoff 2004).

\section{Rückblick und Ausblick auf die Entwicklung der Ölreserven}

Zurückblicken kann man auf die bisherige Entwicklung der Ölfunde, und davon lässt sich - bis zu einem gewissen Grad auf zukünftige Neuentdeckungen schließen. Mengenmäßig wurde das meiste ÖI in den sechziger Jahren gefunden. „Dies ist auch nicht weiter überraschend, da man die großen Felder immer am Anfang und auch mit einfachen Methoden findet $^{56}$ (Schindler/Zittel 2000: 7). Zur Verdeutlichung: Heute sind etwa 42.000 Ölfelder bekannt, doch bereits in einem Prozent dieser Vorkommen sind 75 Prozent des bisher gefundenen Erdöls enthalten. Seit circa zwanzig Jahren übersteigt die Produktion die jährlichen Neufunde. Die Welt ist heute so weitgehend erforsche worden, dass sehr wahrscheinlich alle größeren, leicht abbaubaren Lagerstätten mit konventionellem Öl bereits entdeckt worden sind (Campbell et al 2003: 25). Ungeachtet dessen weist die BP-Studie immer neue Zuwächse aus. In den letzten 20 Jahren haben sich dabei die Reserveangaben um 424,7 Milliarden Barrel erhöht. Das Reservewachstum basiert also darauf, dass "die in bereits produzierenden Feldern insgesamt zu erwartenden Ölmengen dem Produktionsfort- 
schritt folgend laufend höher bewertet" (ebd.: 179) werden. Dass diese Erhöhung kaum auf Neufunde zurückzuführen ist, zeigt das Beispiel der britischen Nordsee: „Mit den ersten 500 Bohrungen wurden $20 \mathrm{~Gb}$ [Gigabarrel = $1 \mathrm{Mrd}$. Barrel] Öl gefunden. Die nächsten 500 Bohrungen erbrachten nur noch einen Zuwachs von etwa $5 \mathrm{~Gb}$, die dritten 500 Bohrungen nur noch $3 \mathrm{~Gb}$. Die 1500 . bis 2000. Bohrungen waren zwar wesentlich erfolgreicher als die ersten Bohrungen - in dem Sinne, dass kaum noch 'trockene' Löcher gebohrt wurden, aber da die Funde deutlich kleiner als in der Frühphase waren, konnten damit nur noch etwa $2 \mathrm{~Gb}$ an Reserven hinzugefügt werden" (ebd.: 26). Um die tatsächliche Entwicklung der Reserven in den Blick zu bekommen, sollte man die zeitliche Entwicklung der Neufunde betrachten, diese Funde aufsummieren und davon dann den bisherigen Ölverbrauch abziehen. „Im Gegensatz zu den öffentlich zugänglichen Reservestatistiken zeigt eine derartige Analyse, dass die Reserven seit etwa 20 Jahren abnehmen. Der Verbrauch kann seit dieser Zeit nicht mehr durch Neufunde ausgeglichen werden" (Schindler/Zittel 2000: 8). Angesichts dessen ist es schwer zu glauben, dass die Internationale Energie Behörde (IEA) in dem in diesem Oktober erschienenen World Energy Outlook sinkende Ölpreise voraussagt. Der derzeitige Ölpreis von ca. 50 Dollar je Barrel soll in Zukunft auf 25 bis 35 Dollar sinken (Wiede 2004). Faith Birol, Organisator und Direktor des World Energy Outlooks sieht nämlich das Hauptproblem in einem "lack of investment in areas where oil reserves are most significant" (Campbell 2004c). Colin Campbell schreibt dazu: "That is possible, but it is kinder to assume that the organisation (the IEA) understands the situation perfectly well yet is under pressure to conceal and confuse, for fear of the panic that might ensue if the truth be told" (ebd.).

\section{Literatur}

BP (2004): Statistical Review of World Energy 2004 , www.bp.com

Campbell C. (2004a): Comparison with BP reserves Estimate, in: Newsletter 44, www.peakoil.net

Campbell C. (2004b): The date of Peak, in: Newsletter 45, www.peakoil.net

Campbell C. (2004c): The IEA: To know or not, in: Newsletter 46, www.peakoil.net

Campbell C./Laherrère J. (1998): The end of cheap oil, in: Scientific American, www.dieoff.org

Campbell C., Liesenborgh F., Schindler J., Zittel W. (2003): Ölwechsel! Das Ende des Erdölzeitalters und die Weichenstellung für die Zukunft, hrsg. vom Global Challenge Network, München 2003.

Darley J. (2004): Colin Campbell Interview, in: Global Public Media, www.globalpublicmedia.com

Friebe R. (2004): Endgültig Schluss mit lustig?, in: FAZ.net, 23. Mai 2004, www.faz.net

Kopytoff V. (2004): Peering into oil's future, 21. März 2004, www.sfgate.com

Kroder T. (2004): Erneute Bewertungspanne verschärft Krise bei Shell, Financial Times Deutschland, 19. März 2004, www.ftd.de

NDR (2004): Ölreserven: Baggern statt bohren, www.ndr.de

Ruppert C. (2004): Colin Campbell on oil, FTW Exclusive Interview, 23. Oktober 2002, www.fromthewilderness.com

Savinar M. (2004): Life after the oil crash deal with reality or reality will deal with you, www.lifeaftertheoilcrash.net

Schempf F. (2004): Simmons hopes he is wrong, in: Petroleum News, Vol. 9, Nr. 31, 1. August 2004, www.petroleumnews.com

Schindler J./Zittel W. (2000): Schriftliche Stellungnahme zu ausgewählten Fragen der Enquete Kommission des Deutschen Bundestages "Nachhaltige Energieversorgung unter den Bedingungen der Globalisierung und der Liberalisierung "zum Thema "Weltweite Entwicklung der Energienachfrage und der Ressourcenverfigbarkeit", Ottobrunn 2000.

Simmons M. (2004): Twilight in the desert: The fading of Saudi Arabia's oil, Washington, www.simmons-intl.com/

Wiede T. (2004): Nicht knapp, aber teuer, in: Handelsblatt, Nr. 209/44, 27. Oktober.

Williams B. (2004): Next big thing: Peak oil, in: Oil \& Gas Joumal, 19.April, www.ogonline.com/ 